\title{
Padronização de um Protocolo Experimental de Treinamento Periodizado em Natação Utilizando Ratos Wistar
}

\author{
Standardization of an Experimental Periodized Training Protocol \\ in Swimming Rats
}

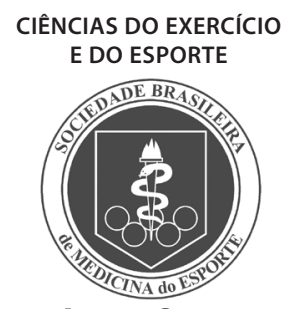

Artigo Original
Gustavo Gomes de Araujo 1,3

Marcelo Papoti, ${ }^{1,2}$

Fúlvia de Barros Manchado-Gobatto ${ }^{1,4}$ Maria Alice Rostom de Mello'

Claudio Alexandre Gobatto

1. Laboratório de Fisiologia Aplicada ao Esporte, Universidade Estadual

Paulista-Unesp, Rio Claro, SP.

2. Universidade Estadual PaulistaUnesp, Presidente Prudente, SP.

3. Faculdades Integradas Einstein de Limeira, Limeira, SP.

4. Universidade Metodista de

Piracicaba, Piracicaba, SP.

\section{Endereço para correspondência:}

Claudio Alexandre Gobatto

Departamento de Educação Física

- Unesp

Av. 24 A, 1.515, Bela Vista

13506-900 - Rio Claro-SP.

E-mail: cgobatto@pq.cnpq.br

\section{RESUMO}

Verificar os efeitos de 12 semanas de treinamento periodizado de natação em ratos sobre os valores de glicogênio muscular (GM) e hepático (GH), capacidade aeróbia (LAn) e anaeróbia (Tlim) e creatina quinase (CK). Foram utilizados 70 ratos da linhagem Wistar com 60 dias, adaptados individualmente ao meio líquido por duas semanas. Os animais foram divididos em grupos: controle $(G C, n=40)$ e periodizado (GP, $n=30$ ); a intensidade do treinamento foi equivalente ao peso corporal do animal (\% do PC). O treinamento (T) para o GP foi dividido em períodos preparatório básico (PPB, seis semanas), específico (PPE, 4,5 semanas) e polimento (PP, 1,5 semana), tendo como estímulos intensidades leve (4\% do PC), moderada ( $5 \%$ do PC), pesada (6\% do PC) e intensa (13\% do PC). Vinte e quatro horas após a adaptação, 10 ratos do GC foram sacrificados e avaliados pelo teste de lactato mínimo para mensuração dos valores de linha de base de GM, GH, CK, LAn e Tlim. Os dez animais restantes do GC e GP foram sacrificados ao final de cada período de T. O treinamento periodizado aumentou a concentração de glicogênio muscular ao final do período de polimento. O glicogênio hepático não se alterou no GC, porém no GP houve redução significativa no início do período específico com elevação no período de polimento. A concentração de CK não se modificou ao final dos PPB, PPE e PP. O LAn reduziu ao longo do período experimental no GC, mas ao final do PP para o GP, o LAn atingiu os mesmos valores do início do treinamento. O Tlim aumentou no PP. Desse modo, pode-se concluir que o treinamento periodizado provocou supercompensação energética ao final da periodização. A capacidade anaeróbia aumentou no PP bem como o LAn, que obteve maiores valores em relação ao GC nesse período.

Palavras-chave: glicogênio, capacidade aeróbia, capacidade anaeróbia e creatina quinase.

\begin{abstract}
The objective of this study was to verify the swimming training periodization responses on aerobic and anaerobic performance, glycogen concentration in muscle $(M)$ and liver $(L)$, and creatine kinase $(C K)$ in rats. Seventy male Wistar rats were randomly separated in two groups: Control Group (CG $n=30)$ and Training Periodization Group (TPG $n=30$ ). All experiments were preceded by 2 weeks of individual adaptation to the water. The TPG was carried out during a period of 12 weeks $(w)$ with frequency of 6 days/W. The training period was subdivided in three specialized series blocks: Preparation ( 6 w), Specific ( $4.5 \mathrm{w}$ ) and Taper $(1.5 \mathrm{w})$. The Lactate Minimun Test (LACM) was adapted to determine the aerobic capacity. Anaerobic performance was evaluated by maximal exhaustion time (Tlim) verified during hyperlactatemia induction phase in the $\mathrm{LACm}$ protocol. Training stimulus was based on intensities corresponding to the LACm: Endurance (END) $1=80 \%$; END2= 100\%; END3 $=120 \%$ and Anaerobic (ANA) 240\% of the LACm. Two-way Anova and Newman-Keuls post-hoc test $(\mathrm{P}<0.05)$ were also used. Aerobic performance was not different from initial training (Preparation: $4.57 \pm 0.24 \%$ of body weigh (bw); Specific: $4.59 \pm 0.44 \% \mathrm{bw}$ ), but at the end of taper, the LACm was higher (Taper: $5.01 \pm 0.71 \%$ bw). The anaerobic parameter (Tlim) was significantly higher at the end of taper $(73 \pm 14 \mathrm{~s})$ when compared to the Preparation (50 $\pm 13 \mathrm{~s})$ and Specific blocks ( $65 \pm 18 \mathrm{~s})$. The $\mathrm{CG}$ reduced the $\mathrm{LACm}$ and anaerobic performance along the experimental period. The glycogen $\mathrm{M}$ increased after taper but CK did not alter during training. Training periodization in rats acted as an important tool to evaluate specific effects of training. This is supported by sensitive responses of the rats along the blocks, based on improvement of aerobic and anaerobic performance as well as glycogen concentration obtained after the taper block.
\end{abstract}

Keywords: glycogen, aerobic capacity, anaerobic capacity and creatine kinase. 


\section{INTRODUÇÃO}

A periodização do treinamento, por possibilitar a maximização da performance e diminuição dos riscos de lesão e supertreinamento ${ }^{(1,2)}$,é uma estratégia frequentemente utilizada por técnicos de vários esportes, tais como a natação ${ }^{(3)}$, corrida ${ }^{(4)}$, treinamento resistido ${ }^{(5)}$, dentre outros.

A principal característica da periodização do treinamento é a inter-relação das variáveis volume e intensidade, ministradas dentro de um período de tempo predefinido, denominado macrociclo. A periodização é a subdivisão do macrociclo em menores períodos, de modo que seja possível a prescrição sistematizada de cada uma das fases do treinamento. De modo geral, ao final da periodização, as cargas são reduzidas objetivando a supercompensação do sistema energético, melhorando assim a performance $e^{(6,7)}$.

Embora exista uma vasta literatura sugerindo diversas maneiras de periodizar o treinamento ${ }^{(8,9)}$, ainda é escasso o embasamento científico que justifique a utilização de determinado modelo de periodização(1), provavelmente pela inviabilidade da implementação de novos métodos de treinamento no âmbito competitivo e, principalmente, pela inexistência de modelos humanos absolutamente bem controlados.

Desse modo, a utilização de modelos animais em programas de treinamento pode ser uma ferramenta importante na manipulação das variáveis volume e intensidade, uma vez que o sistema fisiológico, bem como suas respostas ao exercício, são semelhantes às encontradas em seres humanos ${ }^{(10,11,12,13)}$. Pesquisar métodos de treinamento nesses animais possibilita melhor controle das respostas fisiológicas geradas em diferentes períodos do treinamento por meio de tratamento e análises bioquímicas mais precisas.

Considerando a necessidade de estudos empenhados em investigar os modelos de treinamento periodizado, juntamente com a crescente quantidade de trabalhos que verificam a influência do exercício físico em modelos animais, bem como a viabilidade e bom controle de procedimentos, intervenções e análises laboratoriais ${ }^{(10,13,14)}$, o presente estudo objetivou padronizar um protocolo experimental de treinamento periodizado em natação utilizando ratos Wistar para avaliar precisamente variáveis de performance aeróbia e anaeróbia, substratos energéticos e marcadores bioquímicos ao longo de 12 semanas de treinamento físico. Especificamente, foram dosados, ao final de cada período do treinamento, concentração de glicogênio dos músculos sóleo e gastrocnêmio, glicogênio hepático e concentração de creatina quinase.

\section{MÉTODOS}

\section{Animais}

Foram utilizados 70 ratos da linhagem Wistar com aproximadamente 60 dias de idade. Durante todo o experimento, os animais foram mantidos em gaiolas coletivas, não excedendo cinco ratos por gaiola, em ciclo claro/escuro de 12/12 horas. Todos os animais receberam ração comercial e água ad libitum. Os ratos foram divididos aleatoriamente em grupos: controle ( $G C, n=40)$ e periodizado $(G P, n=30)$. A realização dos experimentos seguiu as resoluções específicas (Lei n 6.638, de 8 de maio de 1979, e Decreto n 24.645, de 10 de julho de 1934).

\section{Adaptação ao meio líquido}

Os ratos foram adaptados ao meio líquido $\left(31 \pm 1^{\circ} \mathrm{C}\right)$ por três semanas, em tanques cilíndricos individuais profundos (120 x 60cm). A partir da 2a semana de adaptação, os animais suportaram sobrecargas equivalentes a $2 \%$ do peso corporal (PC).

\section{Performances anaeróbia e aeróbia}

As performances aeróbia e anaeróbia foram admitidas como intensidade de lactato mínimo (LM) e tempo limite (Tlim). Para determinação dessas variáveis, foi utilizado o protocolo de lactato mínimo (LM) validado por Tegtbur et al. ${ }^{(15)}$ em humanos, aplicado a ratos por Voltarelli et al. ${ }^{(11)}$ e aprimorado para esses animais por de Araujo et al. ${ }^{(13)}$.
O procedimento de avaliação da performance anaeróbia serviu como método para indução da hiperlactacidemia com uma carga correspondente a $13 \%$ do PC. Os animais realizaram dois esforços nessa primeira fase do teste, sendo o primeiro com duração de 30s e, após intervalo de 30s, a realização do segundo esforço na mesma carga até exaustão (Tlim). Amostras sanguíneas $(25 \mu \mathrm{L})$ foram coletadas da extremidade distal da cauda nos minutos 1, 3, 5, 7 e 9 após o Tlim para determinação da concentração pico de lactato $\left(\mathrm{mmol} \cdot \mathrm{L}^{-1}\right)$.

Imediatamente após nove minutos de intervalo passivo, os ratos foram submetidos a um teste incremental para determinação da intensidade de LM (performance aeróbia) correspondente ao equilíbrio entre remoção e produção do lactato. As cargas foram equivalentes a 4,0, 4,5, 5,0, 5,5, 6,0 e 7,0\% do PC de cada animal. Cada estágio do protocolo progressivo foi composto por cinco minutos de duração com intervalo de 30 s para determinação da lactacidemia. A relação obtida entre a concentração de lactato e a carga (porcentagem do peso corporal) foi ajustada por uma equação polinomial de ordem 2, sendo que LM foi considerado a derivada zero desse ajuste ${ }^{(13)}$.

A figura 1 mostra o exemplo gráfico de um rato submetido ao teste progressivo realizado após a indução da lactacidemia e o ajuste polinomial de ordem 2.

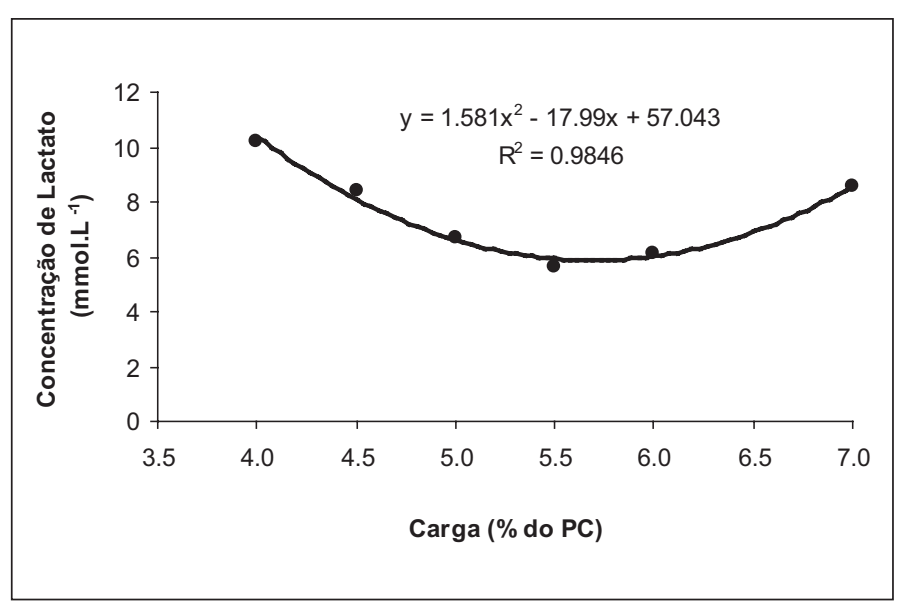

Figura 1. Fase progressiva do teste de lactato mínimo ajustada pela função polinomial de ordem 2. Exemplo retirado de um animal do GP após a adaptação.

\section{Sessões de treinamento: classificação e quantificação dos estímulos aplicados}

Os animais controles (GC) não foram submetidos ao exercício crônico, mas manipulados ao mesmo horário e local dos animais do grupo periodizado (GP).

Os estímulos aplicados durante as sessões de treinamento para o GP foram divididos em séries objetivando o desenvolvimento da aptidão aeróbia e anaeróbia. O treinamento aeróbio foi subdividido em três níveis: Endurance fácil (END1), moderado (END2) e intenso (END 3). O treinamento na intensidade de END1 compreendeu exercícios contínuos com duração-alvo de 60 minutos e realizados em intensidades de $80 \%$ do LM. Foram classificados como séries de END2 os nados contínuos realizados na intensidade correspondente ao LM com duração-alvo de 30 minutos. Os estímulos de END3 envolveram nados intervalados com duração de cinco minutos e um minuto de recuperação, totalizando 15 minutos de exercício com intensidades a 120\% do LM adaptado de Maglisho(16).

Os treinamentos com o objetivo de desenvolver a aptidão anaeróbia (ANA) tiveram esforços intervalados com duração de um minuto e pausa de três minutos, com intensidades entre 220 e 260\% do LM, totalizando cinco minutos de esforço. 
Desse modo, a carga semanal total de treinamento (CST) correspondeu ao somatório dos estímulos de treinamento obtido pelo produto do tempo de esforço (t) e a intensidade relativa (\%) ao LM (figura 2).

$$
\mathbf{C S T}=\left(\mathrm{t}_{1} \cdot \% \mathrm{LM}\right)+\left(\mathrm{t}_{2} \cdot \% \mathrm{LM}\right)+\left(\mathrm{t}_{3} . \% \mathrm{LM}\right)+\ldots
$$

Figura 2. Determinação da carga semanal total de treinamento apresentada em unidades arbitrárias (UA). (Adaptado de Mujika et a ${ }^{\left({ }^{3}\right)}$ ).

\section{Treinamento periodizado}

O treinamento periodizado (GP) apresentou duração total de 12 semanas, sendo dividido em período preparatório básico (PPB), específico (PPE) e polimento (PP).

O PPB teve a duração de seis semanas, sendo composto por estímulos intercalados de END1, END2, END3 e um tempo médio de exercício de 45 min diários. No PPE a duração foi de quatro semanas e meia, com o tempo médio de nado reduzido em 31\%, enquanto que a intensidade foi incrementada em $43 \%$ devido aos esforços realizados na intensidade de ANA. Após essas duas fases, os animais realizaram o PP, com duração total de uma semana e meia, caracterizado pela diminuição progressiva e não linear de $69 \%$ no volume e de $22 \%$ na intensidade em relação aos PPB e PPE, respectivamente (tabela 1 e figura 3).

Tabela 1. Organização do treinamento utilizando as intensidades (END1, END2, END3 e ANA) ao longo de 12 semanas de treinamento periodizado.

\begin{tabular}{c|c|c|c|c|c|c|c}
\hline SEMANAS & Seg & Ter & Qua & Qui & Sex & Sab & FASES \\
\hline $\mathbf{1}$ & TESTE & END2 & END1 & END2 & END1 & END2 & PPB \\
\hline $\mathbf{2}$ & END2 & END1 & END2 & END1 & END2 & END1 & PPB \\
\hline $\mathbf{3}$ & END2 & END1 & END3 & END1 & END2 & END1 & PPB \\
\hline $\mathbf{4}$ & END2 & END3 & END1 & END1 & END2 & END3 & PPB \\
\hline $\mathbf{5}$ & END2 & END3 & END1 & END1 & END2 & END3 & PPB \\
\hline $\mathbf{6}$ & END3 & END2 & END1 & ANA & OFF & TESTE & PPB \\
\hline $\mathbf{7}$ & END2 & ANA & END1 & END3 & END1 & ANA & PPE \\
\hline $\mathbf{8}$ & END2 & ANA & END1 & END3 & END1 & ANA & PPE \\
\hline $\mathbf{9}$ & END2 & ANA & END1 & ANA & END1 & ANA & PPE \\
\hline $\mathbf{1 0}$ & END2 & ANA & END1 & ANA & END1 & ANA & PPE \\
\hline $\mathbf{1 1}$ & END2 & ANA & OFF & TESTE & END2 & END3 & PPE/ PP \\
\hline $\mathbf{1 2}$ & END2 & ANA & END1 & END3 & OFF & TESTE & PP \\
\hline
\end{tabular}

OFF $=$ Sem treino

\section{Desenho experimental e análises bioquímicas}

Dez ratos de cada grupo (GC e GP) foram sacrificados após a adaptação, ao início de cada período da periodização e ao final do período de polimento para determinação dos parâmetros bioquímicos, musculares, hepáticos e de performance aeróbia e anaeróbia (figura 4).

\section{Determinação dos parâmetros sanguíneos e teciduais}

Lactato: Coletas sanguíneas de $25 \mu \mathrm{L}$ foram diluídas em $50 \mu \mathrm{L}$ de fluoreto de sódio a 1\% para análise eletroquímica em lactímetro YSI 1500 sport.

Creatina quinase: Kit MPR3 CKNAC-ativado" (Boehringer Mannheim). O cálculo da concentração de CK (U/L) na amostra foi determinado pela equação $\mathrm{CK}_{\mathrm{p}}=8252 \times \Delta$ absorbância/minuto.

Glicogênio muscular e hepático: Os tecidos foram extraídos e a coloração dos precipitados foi realizada segundo Dubois et al. ${ }^{(17)}$.

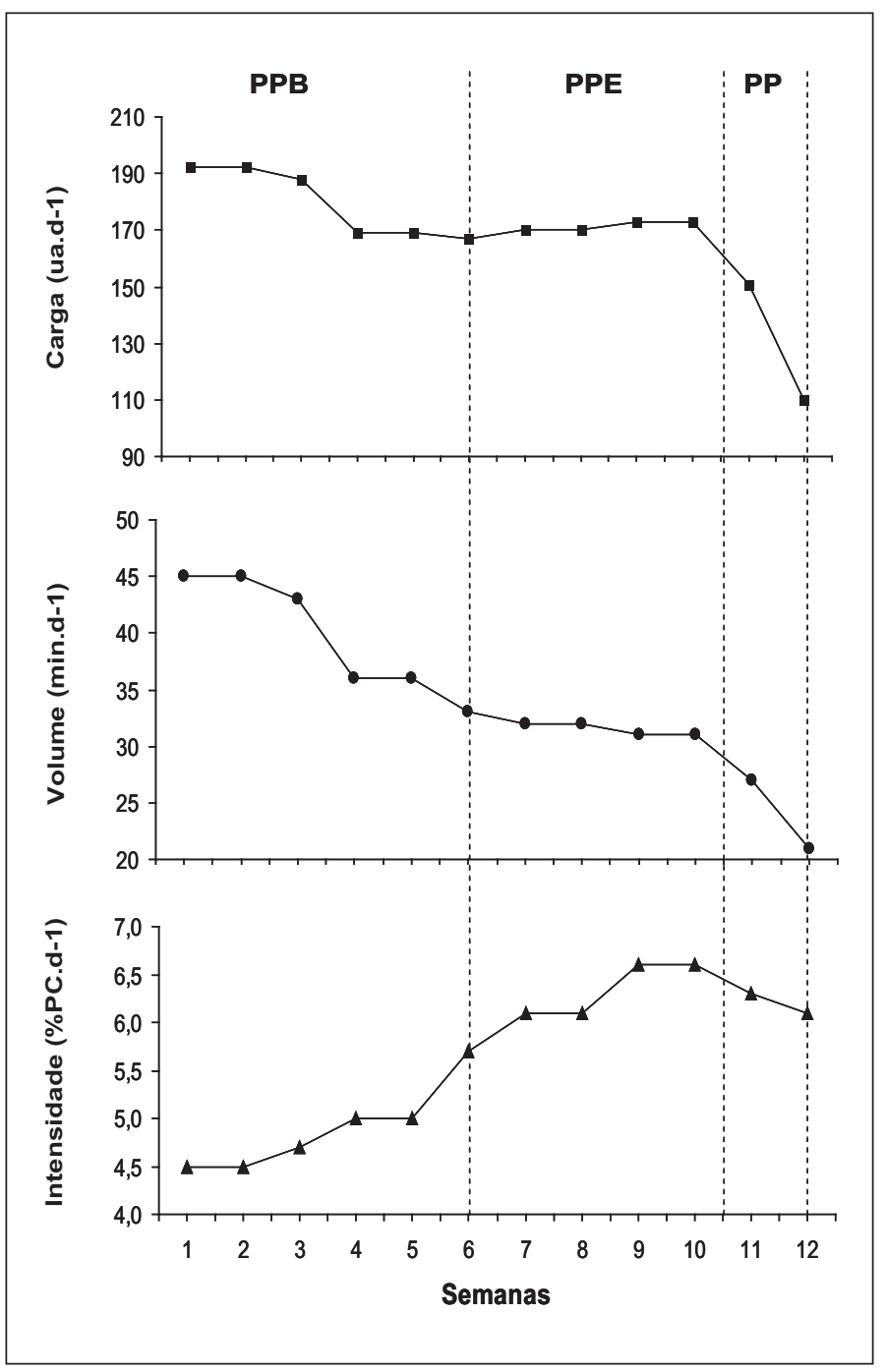

Figura 3. Comportamento teórico das variáveis, volume, intensidade e carga durante os períodos preparatório básico (PPB), específico (PPE) e polimento (PP) ao longo da periodização.

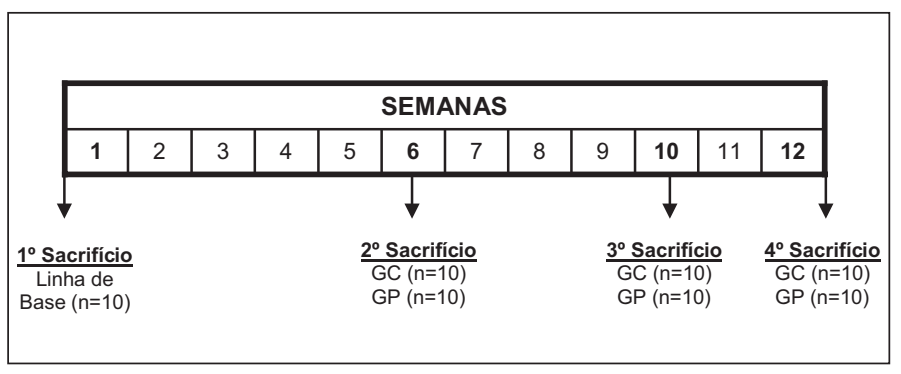

Figura 4. Dias de sacrifício ao longo do treinamento nos protocolos experimentais (GC e GP) no início do experimento e após seis, 10,5 e 12 semanas de treinamento. Os sacrifícios foram realizados $24 \mathrm{~h}$ após a última sessão de treinamento.

\section{Tratamento estatístico}

Os resultados estão expressos em média \pm erro padrão da média. A normalidade e a homogeneidade dos dados foram confirmados com o teste de Shapiro-Wilk. Para os valores dos parâmetros determinados ao longo das fases da periodização foi utilizada a análise de variância ANOVA one-way e, quando necessário, o teste post-hoc de NewmanKeuls. A comparação entre os protocolos de treinamento dos GP e GC foi realizada pelo teste paramétrico $t$ de Student para amostras independentes (Statistica $7.0^{\circ}$ ). O nível de significância foi prefixado em $P<0,05$. 


\section{RESULTADOS}

Os valores médios de peso corporal mensurados no início (I) de cada período de treinamento e ao final (F) do PP estão expressos na tabela 2.

Tabela 2. Valores de peso corporal (g) em todas as fases da periodização nos grupos controle (GC) e periodizado (GP).

\begin{tabular}{c|c|c}
\hline & GC & GP \\
\hline IPPB (linha de base) & $314,1 \pm 4,3$ & $317,1 \pm 3,3$ \\
\hline IPPE & $382,8 \pm 9,4^{a}$ & $392,8 \pm 9,3^{a}$ \\
\hline IPP & $441,2 \pm 9,0^{a, b}$ & $399,3 \pm 16,2^{a, b}$ \\
\hline FPP & $459,1 \pm 26,3^{a, b}, c$ & $418,4 \pm 16,6^{a, b}, c$ \\
\hline
\end{tabular}

a Diferença significativa em relaçăo ao IPPB.

${ }^{b}$ Diferença significativa em relaçăo ao IPPE.

' Diferença significativa em relação ao IPP.

* Diferença significativa em relaçăo ao GP.

\section{Respostas das variáveis volume e intensidade}

O volume de treinamento foi determinado como o somatório total do tempo de esforço para cada intensidade. Não foram verificadas diferenças no volume (min) de treinamento nas intensidades de END1, END2, END3 e ANA do GP com o tempo-alvo (figura 5).

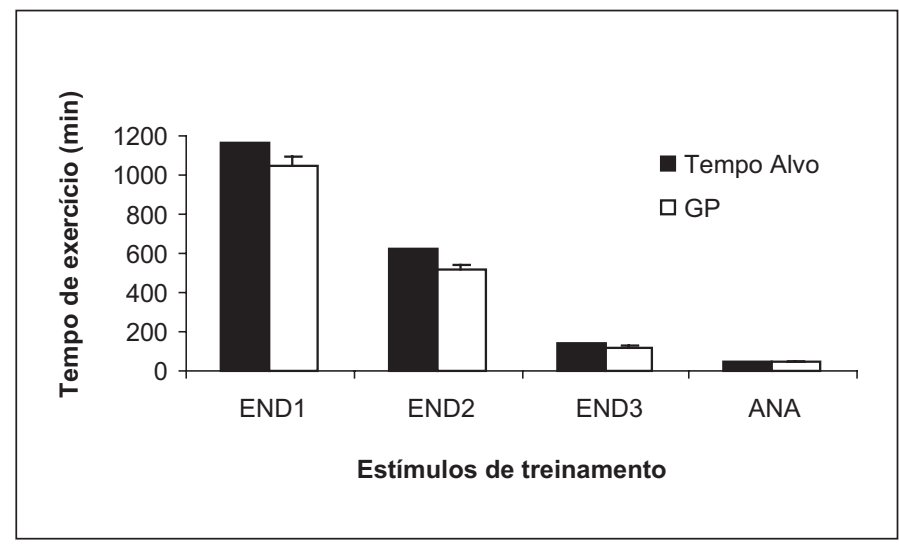

Figura 5. Somatório do tempo ( $\mathrm{min}$ ) total de exercício durante 12 semanas em cada intensidade de treinamento (END1, END2, END3 e ANA) realizado pelo grupo periodizado (GP).

\section{Performances aeróbia e anaeróbia (lactato mínimo e tempo limite) e concentração pico de lactato}

Doze semanas de treinamento periodizado não modificaram o LM do GP; além disso, esses valores não foram diferentes do GC (figura 6A). Já o tempo limite (Tlim) do GP apresentou diferenças significativas com relação ao GC no início (I) do PPE e IPP. Os valores de Tlim do GP ao final (F) do PP foram significativamente superiores aos verificados no GC bem como em relação ao IPPB e IPPE. O Tlim do GC não foi modificado ao longo da periodização experimental (figura 6B).

A concentração pico de lactato não apresentou diferenças significativas entre os grupos GC e GP. Em relação ao comportamento desse metabólito ao longo das fases da periodização, foi constatado que para o GP os valores apresentados nos IPPE, IPP e FPP foram significativamente superiores aos obtidos no IPPB. Além disso, os valores ao FPP foram significativamente superiores ao IPPE. Para o GC as concentrações pico de lactato nas fases IPPE, IPP e FPP foram significativamente superiores às observadas no início da periodização (IPPB) (tabela 3).

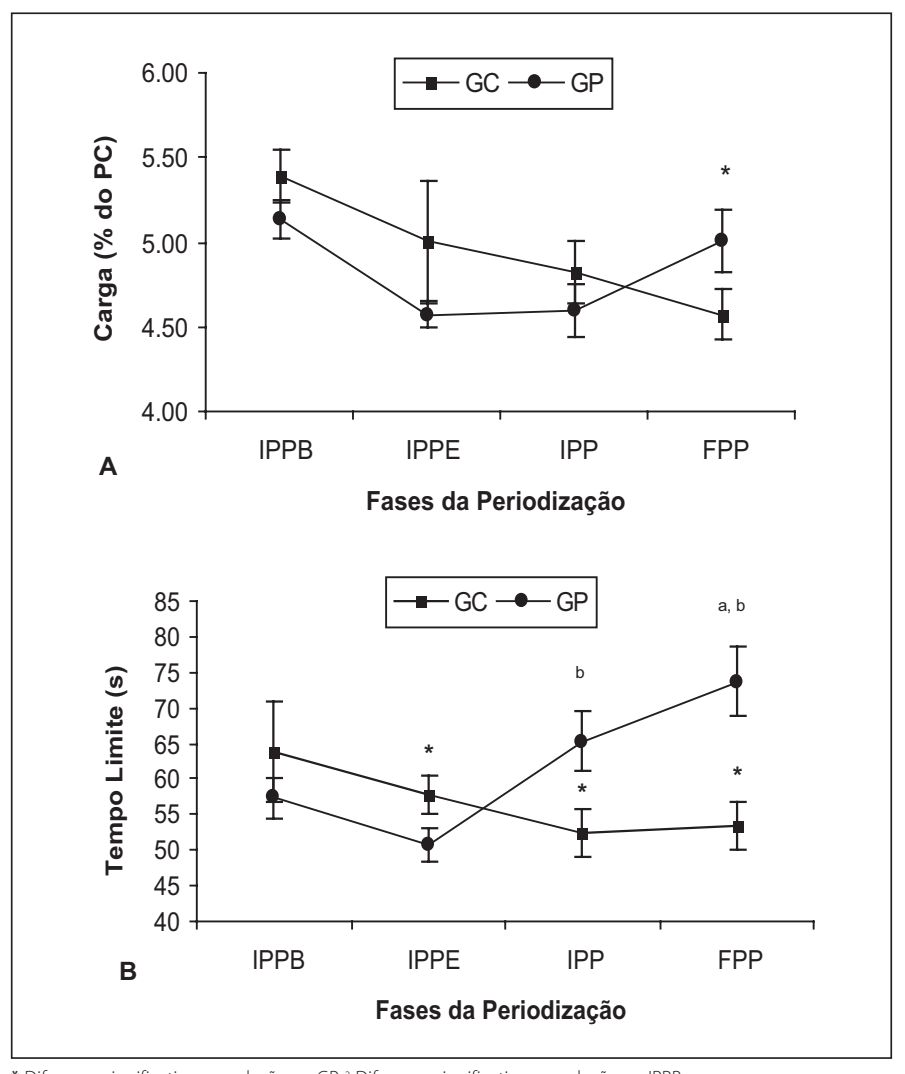

* Diferença significativa em relação ao GP. a Diferença significativa em relação ao IPPB. ${ }^{\text {b }}$ Diferença significativa em relação ao IPPE. c Diferença significativa em relação ao IPP.

Figura 6. Valores de lactato mínimo (A - \% do PC) e tempo limite (B - s) dos grupos controle (GC) e periodizado (GP) durante o início dos períodos preparatório básico (IPPB), específico (IPPE), polimento (IPP) e final do período de polimento (FPP).

Tabela 3. Valores de concentração pico de lactato $\left(m m o l . L^{-1}\right)$ ao final do teste de performance anaeróbia em todas as fases da periodização nos grupos controle (GC) e periodizado (GP)

\begin{tabular}{c|c|c}
\hline & GC & GP \\
\hline IPPB (linha de base) & $5,48 \pm 1,37^{*}$ & $6,65 \pm 0,95$ \\
\hline IPPE & $8,69 \pm 0,68^{\mathrm{a}}$ & $7,49 \pm 1,42^{\mathrm{a}}$ \\
\hline IPP & $8,25 \pm 1,18^{\mathrm{a}}$ & $8,26 \pm 1,15^{\mathrm{a}}$ \\
\hline FPP & $9,13 \pm 0,84^{\mathrm{a}}$ & $8,89 \pm 1,05^{\mathrm{a}} \mathrm{b}$ \\
\hline
\end{tabular}

* Diferença significativa em relaçăo ao GP.

${ }^{a}$ Diferença significativa em relaçăo ao IPPB.

b Diferença significativa em relação ao IPPE.

\section{Concentração de glicogênio muscular (sóleo e gastrocnê- mio) e hepático}

A concentração de glicogênio do gastrocnêmio no GP reduziu-se no IPPE em relação aos outros períodos. Apesar disso, o glicogênio do gastrocnêmio aumentou no IPP, atingindo valores significativamente superiores no FPP (figura 7A).

Diferentemente, no músculo sóleo, os valores de glicogênio obtidos no GP não foram modificados ao longo da periodização, porém, foram maiores que o GC no FPP (figura 7B). Em relação ao GC, as concentrações de glicogênio do músculo gastrocnêmio e sóleo não foram diferentes em nenhuma das fases da periodização, apresentando valores significantemente reduzidos no FPP em relação ao GP (figura 7A-B). As concentrações hepáticas de glicogênio no GP nas fases IPP e FPP foram maiores com relação ao IPPE. Além disso, no IPPE as concentrações hepáticas de glicogênio diminuíram significativamente em relação ao GC, que se manteve com valores próximos nos IPP e FPP (figura 7C). 


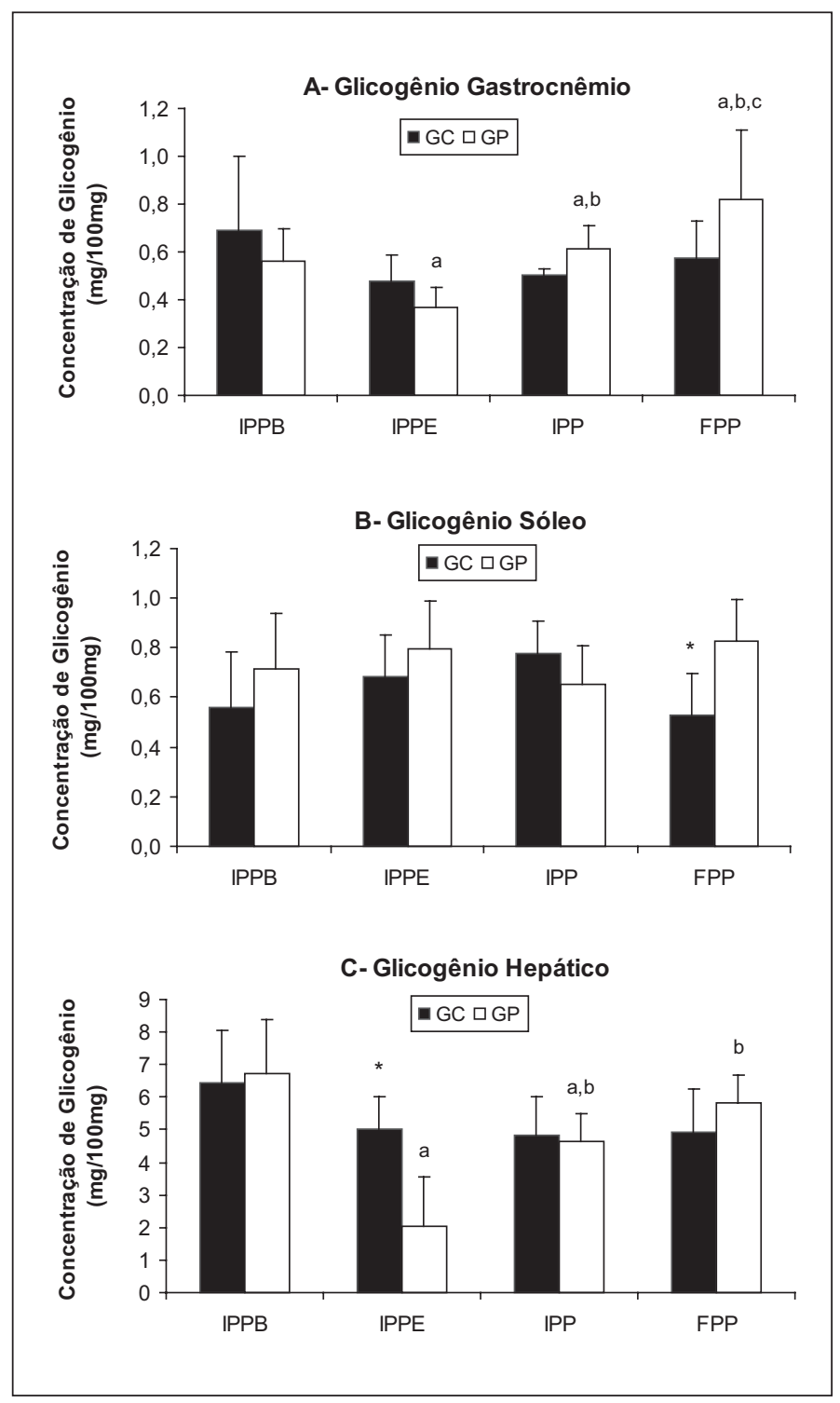

Figura 7. Concentração de glicogênio do gastrocnêmio (A), sóleo (B) e do tecido hepático (C) nos períodos IPPB, IPPE, IPP e FPP. * Diferença significativa em relação ao GP no mesmo período. ${ }^{a}$ Diferença significativa em relação ao IPPB. ${ }^{b}$ Diferença significativa em relação ao IPPE. ${ }^{c}$ Diferença significativa em relação ao IPP.

\section{Creatina quinase (CK)}

Não foram verificadas diferenças ao longo da periodização nos valores séricos de CK nem mesmo entre os GP e GC (figura 8).

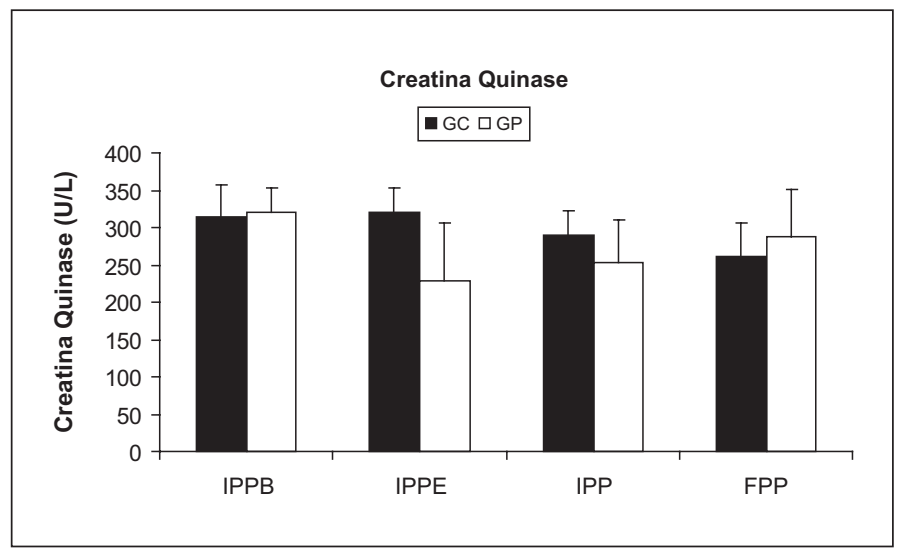

Figura 8. Valores absolutos de CK (U/L) ao longo da periodização nos diferentes períodos.

\section{DISCUSSÃO}

O modelo de periodização do treinamento padronizado para ratos no presente estudo foi baseado no modelo clássico semelhante ao proposto por Matveev ${ }^{(8)}$ para humanos. A distribuição dos estímulos mensurados pelo volume de treinamento foi reprodutível em todas as intensidades da periodização experimental (figura 2).

Os treinamentos na intensidade de END1 objetivaram a restauração parcial dos teores de glicogênio intramuscular, sendo as sessões de END1 realizadas principalmente após séries de esforços intensos, como anteriormente sugerido na literatura ${ }^{(4,18)}$. Entre as séries utilizadas para desenvolvimento da capacidade aeróbia, o treinamento na intensidade correspondente ao limiar anaeróbio (END2) possibilitou manter a mais alta concentração de lactato sem proporcionar acúmulo progressivo da lactacidemia ao longo da sessão. As séries END3 foram intensidades acima do limiar anaeróbio aplicadas com objetivo de desenvolver a potência aeróbia. Os estímulos em ANA objetivaram desenvolver a capacidade anaeróbia com treinamentos intensos suportando períodos com alta produção de lactato(16).

Apesar de as taxas de depleção e restauração dos estoques de glicogênio para cada intensidade não terem sido mensuradas, é provável que os estímulos de treinamento tenham alcançado suas finalidades, demonstradas principalmente pelas diferentes concentrações desse polímero em resposta às diferentes características de cada período de treinamento (figura 4).

O tempo de nado até a exaustão (Tlim), que foi admitido no presente estudo como parâmetro de performance anaeróbia, foi significativamente alterado ao longo das fases do treinamento, especialmente no IPP, que foi o período de treinamento no qual estiveram contidos os estímulos de treinamento mais intensos, bem como as maiores concentrações de glicogênio. A redução do Tlim após o PPB pode ter ocorrido devido ao elevado volume de treinamento aeróbio que, por sua vez, pode levar à estagnação e comprometimento do desempenho anaeróbio, refletido no presente estudo com a redução da oferta glicolítica de energia e diminuição do Tlim no IPPE ${ }^{(19)}$. Além disso, Fitts et al. ${ }^{(20)}$ relataram significativas reduções de 15\% nas fibras musculares do tipo lla de nadadores após 10 dias de treinamento com elevado volume.

Desse modo, o esperado seria que a capacidade aeróbia determinada pelo LM sofresse aumentos significativos ao longo da periodização, principalmente após o período de elevado volume de treinamento aeróbio (PPB). No entanto, não foram observadas diferenças na resposta aeróbia ao longo do treinamento (figura 3A). Carter et al. ${ }^{(21)}$ demonstraram que o teste de LM não é valido para determinar os efeitos do treinamento em humanos devido a sua reduzida sensibilidade aos ganhos aeróbios, sugerindo que outros protocolos de avaliação possam ser mais precisos. Por outro lado Voltarelli et al.(22) encontraram, após quatro e oito semanas de treinamento de endurance em natação, positivas modificações na capacidade aeróbia de ratos determinada pelo teste de LM. No presente estudo, a queda da capacidade aeróbia ao longo do tempo parece ser um processo natural da espécie, porém, o treinamento periodizado, mesmo não melhorando o LM, atenuou essa redução em relação ao GC.

A concentração pico de lactato foi utilizada como indicador da capacidade produtora de lactato ao longo do treinamento. As maiores diferenças foram observadas a partir do IPPE em ambos os grupos, quando comparados com o IPPB. Contudo, a capacidade produtora de lactato do GC também aumentou significativamente após esse período, descartando assim a hipótese de alterações em função do treinamento. Medbø e Toska ${ }^{(23)}$ demonstraram que a concentração sanguínea de lactato não reflete a produção energética real oriunda do glicogênio 
muscular. No entanto, outros pesquisadores verificaram que a formação de lactato pode estar relacionada com a taxa de depleção energética pelo glicogênio muscular ${ }^{24,25)}$, podendo inclusive ser utilizado ao longo do treinamento como marcador de supertreinamento ${ }^{(24)}$. No presente estudo, devido à produção de lactato ter aumentado em ambos os grupos, uma justificativa para esse efeito seria que o aumento da lactacidemia após as seis semanas iniciais de treinamento tenha simplesmente ocorrido em função do desenvolvimento maturacional da espécie e/ou aumento do peso corporal total, ocasionando aumento da massa muscular e, consequentemente, maior capacidade de produção lactacidêmica natura|(26).

A CK é uma enzima amplamente utilizada como biomarcador de estresse e alteração na atividade muscular. A elevação sérica da CK é atribuída a danos teciduais, podendo resultar em aumento na permeabilidade da membrana celular, entre outros fatores, devido à peroxidação lipídica(24). Tem sido verificado que as concentrações circulantes de CK são alteradas conforme as mudanças nas variáveis volume e intensidade de treinamento ${ }^{(24)}$. No entanto, o período de polimento é a principal fase na qual há melhor recuperação muscular, diminuindo os valores séricos dessa enzima em relação aos outros períodos ${ }^{(27)}$. No presente estudo, não foram constatadas variações significativas nas concentrações séricas dessa enzima ao longo das fases da periodização nos GP e GC(28). Essa resposta sugere que a manipulação das variáveis volume e intensidade ao longo das 12 semanas de periodização não resultou em lesão tecidual significativa, corroborando estudos que demonstram que o treinamento periodizado, além de maximizar a performance no momento desejado, também proporciona a diminuição dos riscos de supertreinamento ${ }^{(1,2)}$.

O polimento é o final do período de treinamento que antecede a competição-alvo ${ }^{(6,29)}$. No presente estudo simulamos esse período da periodização em animais. Segundo a literatura, nesse período a carga de treinamento é reduzida, proporcionando recuperação fisiológica e psicológica do atleta na tentativa de promover aumento do rendimento no momento desejado ${ }^{(27)}$. No entanto, não se sabe qual o modelo ideal de polimento a ser aplicado, existindo divergências na literatura a respeito dos métodos a serem utilizados ${ }^{(7)}$. No presente estudo, o polimento teve redução de aproximadamente $69 \%$ no volume e $22 \%$ na intensidade, desencadeando o processo de supercompensação dos substratos energéticos demonstrado com as maiores concentrações de glicogênio dos músculos sóleo e gastrocnêmio, bem como maiores valores no tempo de exaustão (Tlim), caracterizando maior performance aeróbia e anaeróbia dos animais.

Dessa maneira, os resultados obtidos demonstraram que o modelo de periodização do treinamento na fisiologia experimental pode ser aplicado de maneira similar à executada em humanos. Cabe ressaltar que o modelo proposto pode ser capaz de equacionar dúvidas provenientes da aplicação da periodização em esporte de alto rendimento, devido à possibilidade de maior controle extrínseco, bem como manipulações invasivas nesses animais. A supercompensação dos substratos energéticos, bem como a manutenção da capacidade aeróbia e aumento do tempo de exaustão ao final do período do polimento, reflete a sensibilidade do treinamento aos estímulos aplicados e o sucesso na obtenção do pico de performance em um modelo experimental de treinamento periodizado.

\section{AGRADECIMENTOS}

Fundação de Amparo a Pesquisa do Estado de São Paulo (Fapesp proc. 04/01205-6; 06/58411-2) e Conselho Nacional de Desenvolvimento Científico e Tecnológico (CNPq proc. 301601/2006-2).

Todos os autores declararam não haver qualquer potencial conflito de interesses referente a este artigo.

\section{REFERÊNCIAS BIBLIOGRÁFICAS}

1. Fry RW, Morton AR, Garcia-Webb P, Crawford GPM, Keast D. Biological responses to overload training in endurance sports. Eur J Appl Physiol 1992;64:335-44.

2. Rowbottom DG. Periodização do treinamento. In: Garret Jr WE, Kirkendall DT. A ciência do exercício e dos esportes. Artmed, 2003;531.

3. Mujika I, Chatard JC, Busso T, Geyssant A, Barale F, Lacoste L. Effects of training on performance in competitive swimming. Can J Appl Physiol 1995;20:395-406.

4. Martin DE, Coe PN. Developing running with periodization of training. Champaign: Human Kinetics, 1997;167-252.

5. Kramer WJ, Nindl BC, Ratamess NA, Gotshalk LA, Volek JS, Fleck SJ, et al. Changes in muscle hypertrophy in women with periodized resistance training. Med Sci Sports Exerc 2004:36:697-708.

6. Mujika I, Padilla S. Scientific bases for precompetition tapering strategies. Med Sci Sports Exerc 2003;35:1182-7.

7. Thomas L, Busso T. A theoretical study of taper characteristics to optimize performance. Med Sci Sports Exerc 2005;37:1615-21.

8. Matveev LP. Treino desportivo: metodologia e planejamento. São Paulo: Phorte, 1997.

9. Bompa TO. Periodização: teoria e metodologia do treinamento. São Paulo: Phorte, 1999.

10. Gobatto CA, de Mello MA, Sibuya CY, de Azevedo JR, dos Santos LA, Kokubun E. Maximal lactate steady state in rats submitted to swimming exercise. Comp Biochem Physiol A Mol Integr Physiol 2001;130:21-7.

11. Voltarelli FA, Gobatto CA, Mello MAR. Determination of aerobic threashold in rats rising the lactate minimum test. Braz J Med Biol 2002;35:1389-94.

12. Manchado FB, Gobatto CA, Contarteze RVL, Papoti M, Mello, MAR. Maximal lactate steady state in running rats. J Exerc Physiol online 2005;8:29-35.

13. de Araujo GG, Papoti M, Manchado FB, Mello MA, Gobatto CA. Protocols for hyperlactatemia induction in the lactate minimum test adapted to swimming rats. Comp Biochem Physiol A Mol Integr Physiol 2007;148:888-92.

14. Contarteze RV, Manchado FB, Gobatto CA, Mello MA. Stress biomarkers in rats submitted to swimming and treadmill running exercises. Comp Biochem Physiol A Mol Integr Physiol 2008;151:415-22.

15. Tegtbur U, Busse MW, Braumann KM. Estimation of an individual equilibrium between lactate production and catabolism during exercise. Med Sci Sports Exerc 1993;25:620-7.
16. Maglisho EW. Nadando ainda mais rápido. São Paulo: Manole, 1999.

17. Dubois B, Gilles KA, Hamilton JK, Rebers PA. Colorimetric method for determination of sugar and related substances. Anal Chem 1956;28:350-6.

18. Baldari C, Videira M, Madeira F, Sergio J, Guidetti L. Blood lactate removal during recovery at various intensities below the individual anaerobic threshold in triathletes. J Sports Med Phys Fitness 2005;45:460-6

19. Costill DL, Thomas R, Robergs RA, Pascoe D, Lambert C, Barr S, et al. Adaptations to swimming training: influence of training volume. Med Sci Sports Exerc 1991;23:371-7.

20. Fitts RH, Costill DL, Gardetto PR. Effect of swim exercise training on human muscle fiber function. J Appl Physiol 1989;66:465-75.

21. Carter $\mathrm{H}$, Jones AM, Doust JH. Effect of 6 weeks of endurance training on the lactate minimum speed. J Sports Sci 1999;17:957-67.

22. Voltarelli FA, Mello MAR, Gobatto CA. Limiar anaeróbio determinado pelo teste do lactato mínimo em ratos: efeito dos estoques de glicogênio muscular e do treinamento físico. Revista Portuguesa de Ciências do Desporto 2004;4:16-25

23. Medbø Jl, Toska K. Lactate release, concentration in blood, and apparent distribution volume after intense bicycling. Jpn J Physiol 2001;51:303-12.

24. Halson S, Jeukendrup A. Does overtraining exist? An analysis of overreaching and overtraining research. Sports Med 2004;34:967-81.

25. Shulman RG. Glycogen turnover forms lactate during exercise. Exerc Sport Sci Rev 2005;33:157-62.

26. Lodder MA, de Haan A, Sargeant AJ. Effect of growth on efficiency and fatigue in extensor digitorum longus muscle of the rat. Eur J Appl Physiol Occup Physiol 1994;69:429-34.

27. Mujika I, Padilla S, Pyne D, Busso T. Physiological changes associated with the pre-event taper in athletes. Sports Med 2004;34:891-927.

28. Kanter MM, Lesmes GR, Kaminsky LA, La Ham-Saeger J, Nequin ND. Serum creatine kinase and lactate dehydrogenase changes following an eighty kilometer race. Relationship to lipid peroxidation. Eur J Appl Physiol Occup Physiol 1988;57:60-3

29. Papoti M, Martins LE, Cunha SA, Zagatto AM, Gobatto CA. Effects of taper on swimming force and swimmer performance after an experimental ten-week training program. J Strength Cond Res 2007:21:538-42. 